\title{
Adaptive Control of Small Outboard-Powered Boats for Survey Applications
}

\author{
A. D. Fisher \\ Florida Atlantic University \\ 101 North Beach Road \\ Dania Beach, FL 33004, USA \\ afishe14@fau.edu
}

\author{
J .H. VanZwieten Jr. \\ Florida Atlantic University \\ 101 North Beach Road \\ Dania Beach, FL 33004, USA \\ jvanzwi@,fau.edu
}

\author{
T.S. VanZwieten \\ NASA/Marshall Space Flight Center \\ Mail Code EV42 \\ Huntsville, AL 35812 USA \\ tannen.s.vanzwieten@nasa.gov
}

\begin{abstract}
Four autopilot controllers have been developed in this work that can both hold a desired heading and follow a straight line. These PID, adaptive PID, neuro-adaptive, and adaptive augmenting control algorithms have all been implemented into a numerical simulation of a 33-foot center console vessel with wind, waves, and current disturbances acting in the perpendicular (across-track) direction of the boat's desired trajectory. Each controller is tested for its ability to follow a desired heading in the presence of these disturbances and then to follow a straight line at two different throttle settings for the same disturbances. These controllers were tuned for an input thrust of $2000 \mathrm{~N}$ and all four controllers showed good performance with none of the controllers significantly outperforming the others when holding a constant heading and following a straight line at this engine thrust. Each controller was then tested for a reduced engine thrust of $1200 \mathrm{~N}$ per engine where each of the three adaptive controllers reduced heading error and across-track error by approximately $50 \%$ after a 300 second tuning period when compared to the fixed gain PID, showing that significant robustness to changes in throttle setting was gained by using an adaptive algorithm.
\end{abstract}

Keywords-Autopilot, Adaptive Control, Neuro-adaptive, Adaptive Augmentation, Adaptive PID, Outboard Motor Control

\section{INTRODUCTION}

Conducting surveys of the marine environment is one of the most important aspects of ocean research. Many of these surveys require a specific area to be studied, which is typically done by performing a series of parallel straight-line passes separated by a distance required by the sensor for the desired resolution. This is especially true when monitoring a certain location to determine the change in the environmental conditions, marine habitat, or sea life over time.

Many surveys are being done on progressively smaller vessels as sensors decrease in size resulting from technological advances. While this has the benefit of reducing the cost of these surveys, the smaller vessels increase the complexity of control design. Smaller vessels are typically controlled using only rear propulsion systems without the aid of bow or stern thrusters. This lack of control authority makes it difficult to reject wind, wave, and current disturbances, as the sway motion of these vessels cannot be directly controlled. If manual control is used for surveys that require minimal across-track error it is necessary for a skilled captain to continually compensate for these disturbances, which requires him or her to constantly monitor the navigational instruments while remaining aware of the vessel's surrounding. The goal of this project is to automatically mitigate these disturbances to allow the vessel to follow a given trajectory or path. The design will focus on the implementation of various adaptive controllers that have the capability of self-tuning in response to changes in the weather conditions, throttle setting, or vessel dynamics. These algorithms are then compared against the commonly used fixed-gain PID controller.

There currently exist multiple commercially available autopilots that steer the vessel along a given heading or course [1]. These autopilots use increasingly complex algorithms which perform a number of tasks, including course following and navigating to a waypoint [2], [3], [4]. A drawback of these current autopilots is that they are predominantly designed for navigation in light weather conditions [5]. The adaptive control methodologies presented in this paper includes control gains which adapt on-line, allowing the controllers to tune themselves in accordance with the current weather conditions or when towing instruments changes the dynamics of the vessel. These controllers can either hold the vessel's heading near the desired heading or follow a predetermined straight line with minimal cross track error while adapting the controller's gains to increase performance.

This paper is organized as follows: The testing platforms used for development and implementation of the controllers is addressed in Section 2. Section 3 presents all of the adaptive and fixed-gain controllers that have been developed, and Section 4 quantifies the performance of these controllers through simulation. Finally, the conclusions are given in Section 5.

\section{TESTING PlatFormS}

For the development and evaluation of the constant heading and path-following controllers, the validated numerical simulation presented in [6] was utilized. Within the simulation, there are 17 user defined parameters that define the physical dimensions of the vessel [6]. These parameters are set to match those of FAU's Center for Ocean Energy Technology's research vessel, the RV Ocean Power. The RV Ocean Power is a $10 \mathrm{~m}$ (33 foot) center console vessel with $3.3 \mathrm{~m}$ ( 11 foot) 
beam and $0.75 \mathrm{~m}$ ( 2.5 foot $)$ draft. The vessel in the original simulation had dual inboard motors which steered using rudders, and the simulation was modified to represent the dual outboard motors on the current test vessel.

To evaluate the developed controllers, a desired heading or path is used. For this application, a constant heading (straight path) was set. The desired path used in this paper dictates that the vessel shall travel due east while mitigating environmental disturbances acting on it in the northward direction. This was done to mimic the conditions an actual vessel may experience in the Gulf Stream off Florida's southeast coast where the current flows in the northerly direction. The Easterly path was chosen because one of the test vessel's primary uses is collecting ADCP data across the Gulf Stream.

In the simulation, various environmental conditions can be set to act on the vessel as well. For this paper, the following conditions were set: the wind is modeled as a Davenport spectrum [7] with mean wind speed of $10 \mathrm{~m} / \mathrm{s}$ at $10 \mathrm{~m}$ above sea level, the current is a constant $1.5 \mathrm{~m} / \mathrm{s}$, and the significant wave height is $0.5 \mathrm{~m}$ modeled using a spectrum developed in [8]. It should be noted that the wave forces modeled in this simulation only include those induced by the horizontal orbital velocities from the waves [6].

Thrust was not taken as a control variable for the work done in this paper. Rather, each control was tuned based on an engine's thrust at a constant value of $2000 \mathrm{~N}$. At this level of thrust, a forward speed of $3.9 \mathrm{~m} / \mathrm{s}$ is produced when the vessel is operating in still environmental conditions. This was configured to replicate a survey where a captain could set the thrust and engage the autopilot to steer to the desired path. Additionally, a limit was put on the engines to only turn $\pm 35^{\circ}$, as this was the physical limit of the boat's engines; and a turning rate limit of $10^{\circ}$ per second was also put on the simulated vessel.

The simulation allows for a controller to be developed, with the controller's inputs being the boat's heading and heading rate as well as northward (across-track) velocity and position; with the controller the setting the engine angles. The engine angles are combined with the environmental effects stated above and fed through dynamics calculations that find the boat's new velocities, positions, and headings, which then are fed back to the controller.

\section{CONTROL DESIGNS}

The controllers in this paper have two functions: holding the vessel at a fixed heading and following a predetermined path. Of these two functions, following a straight path is the primary focus of this paper. To accomplish the primary goal, the across-track error is used to set the desired heading so that it can be indirectly minimized if the actual heading can be approximately matched to the desired heading. Thus, there are two layers used by the controls methodology in this autopilot: a first control algorithm uses across-track displacement and velocity to calculate the desired heading and a second control algorithm works to match the actual heading to the desired heading.

For this project, a standard PID controller is used in the first control layer to calculate the desired heading and the desired rotation rate on-line, while various fixed-gain and adaptive controllers are used in the second control layer to minimize heading error and in some cases the rotation rate. The first layer PID controller that is used for each simulation is presented first, followed by several options for a second layer controller.

\section{A. First Layer PID Controller}

As stated earlier, the primary objective of the control scheme is for the boat to go due east in the presence of environmental forces pushing it off course. Thus, with north being considered $0^{\circ}$, the desired heading is $90^{\circ}$ plus compensation for the error in the north (across-track) direction. The following relations are used to define the desired orientation and rotation rate:

$$
\begin{aligned}
& \psi_{d}=K_{P}^{a} N_{e}+K_{I}^{a} \int N_{e}+K_{D}^{a} \dot{N}_{e}+90^{\circ} \\
& \dot{\psi}_{d}=N_{e} * K_{I}^{a}+\dot{N}_{e} * K_{P}^{a}
\end{aligned},
$$

where $N_{e}=N_{a c t u a l}-N_{d}$ and $\dot{N}_{e}=\dot{N}_{a c t u a l}-\dot{N}_{d}$, with $N_{d}=0$ and $\dot{N}_{d}=0 . N_{d}$ is defined as the desired northward displacement (0) and $N_{a c t}$ is the actual north position. The desired rotation rate $\dot{\psi}_{d}$ is approximated from the derivative of the desired heading, $\psi_{d}$, with the $\ddot{N}_{e}$ term removed as the vessel does not currently have a way of measuring northward acceleration.

In this primary controller, the gains are shown in Table I. These gains were found through iterative tuning to minimize across-track error while also keeping gains low enough to maintain a control command within the vessel's achievable range..

TABLE I: FIRST LAYER PID CONTROLLER GAINS

\begin{tabular}{|c|c|c|}
\hline$K_{P}^{a}$ & $K_{I}^{a}$ & $K_{D}^{a}$ \\
\hline $0.03(\mathrm{rad} / \mathrm{m})$ & $0.0007(\mathrm{rad} /(\mathrm{m}-\mathrm{s}))$ & $0.02(\mathrm{rad}-\mathrm{s} / \mathrm{m})$ \\
\hline
\end{tabular}

As noted above, the first layer fixed-gain PID control law given in (1) was used in all four control schemes with the same gains to determine the boat's desired heading and rotation rate. The schemes differed in the implementation of the second 
layer's control law. This standard PID controller calculates the desired heading which serves as an input to the four secondary controllers described below.

\section{B. Second Layer PID Controller}

A PID controller is used as one of the second layer controllers because this is a well-known algorithm that is standard for many applications. It will be used here as a baseline controller for the adaptive control algorithms to be compared against. The desired heading and rotation rate calculated from the first layer controller along with the actual heading and rotation rate are inputs to the second layer controller. The output of the second-layer controller is the commanded engine angle that is designed to minimize both the heading and rate errors. The commanded engine angle is given by:

$$
u_{\psi}=K_{P}^{b} e_{\psi}+K_{I}^{b} \int e_{\psi}+K_{D}^{b} \dot{e}_{\psi},
$$

where $e_{\psi}=\psi_{\text {actual }}-\psi_{d}$ is the heading error and its approximated derivative is $\dot{e}_{\psi}=\dot{\psi}_{\text {actual }}-\dot{\psi}_{d}$, with the desired heading and rotation rate calculated in the first layer. For this controller, the gains used for the simulation are shown in Table II. These gains were found using iterative tuning that minimized the initial heading overshoot with fast convergence, while balancing this with the desire to keep the commanded engine angle well within its achievable range.

TABLE I: SECOND LAYER PID CONTROLLER GAINS
\begin{tabular}{|c|c|c|}
\hline$K_{P}^{b}$ & $K_{I}^{b}$ & $K_{D}^{b}$ \\
\hline 0.5 & $0.006\left(\mathrm{~s}^{\wedge}-1\right)$ & $2(\mathrm{~s})$ \\
\hline
\end{tabular}

\section{Adaptive PID Controller}

The structure of the adaptive PID is the same as its fixed gain counterpart given by equation (2), but here the gains will adapt on-line. The structure of the adaptive PID the same as its fixed-gain counterpart with separate proportional, integral, and derivative terms that sum up to make the control signal. The derivative of the three adaptive PID gains are calculated by

$$
\dot{g}_{P}=-\Gamma_{1} e_{\psi}{ }^{2}+\sigma_{1} g_{P} \quad \dot{g}_{I}=-\Gamma_{2} e_{\psi} \int e_{\psi}-\sigma_{2} g_{I} \quad \dot{g}_{D}=-\Gamma_{3} e_{\psi} \dot{e}_{\psi}-\sigma_{3} g_{D},
$$

where $\Gamma_{1,2,3}$ are gains chosen by the designer to control the rate of adaptation for each term. The second term in the adaptive update law, $\sigma_{1,2,3}$, is called the sigma modification term and is used to prevent wind-up. The adaptive PID gains are calculated by integrating (3), and each gain is given an initial value of 0 . The engine angle is then calculated as

$$
u_{\psi}=g_{P} e_{\psi}+g_{I} \int e_{\psi} d t+g_{D} \dot{e}_{\psi}
$$

The adaptation rate values, $\Gamma_{1,2,3}$, and values for $\sigma_{1,2,3}$ used in the simulation are found in Table III. These values were determined iteratively for fast adaptation and good tracking of the desired trajectory over time, while keeping gains low enough to minimize actuator saturation.

TABLE III: ADAPTIVE CONTROLLER GAINS AND LEAKAGE TERMS
\begin{tabular}{|c|c|c|c|} 
& Proportional & Integral & Derivative \\
\hline Gamma & -0.2 & -.003 & -12 \\
\hline Sigma & 0.01 & 0.01 & 0.02 \\
\hline
\end{tabular}

\section{Single Layer Neuro-Adaptive Controller}

The second adaptive methodology utilizes a single layer neural network controller which uses radial basis functions. As before, the goal is to minimize heading error. To create the neural network, a regressor vector $\Phi$ is selected to be

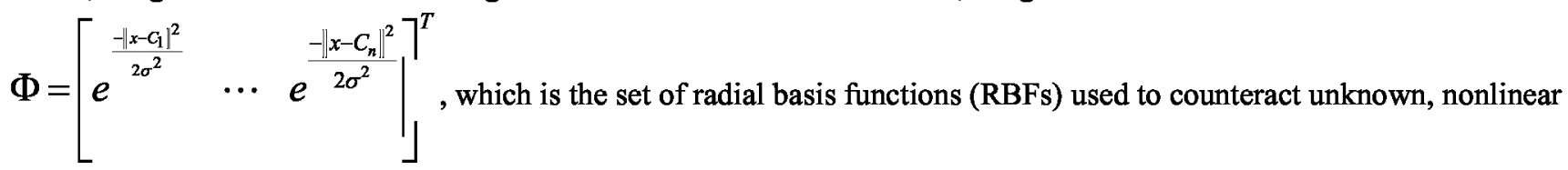

aspects of the plant. Radial basis functions are chosen because they act as universal approximators [7]. The values of function centers $C_{1} \ldots C_{n}$ and $\sigma$ are design parameters. Here, the values of the $C_{1} \ldots C_{n}$ centers were set to ensure constant spacing between functions and the value of $\sigma$ was found using the difference between RBF centers divided by $2 \sqrt{\log (2)}$ to ensure the Gaussian functions (RBFs) cross at their vertical midpoints.

In this controls layer, 22 neurons are used $(n=22)$. Following the architecture suggested by [8], the commanded engine angle is calculated by 


$$
u_{\psi}=\hat{K}_{e}^{T} e_{\psi}-\hat{\Theta}^{T} \Phi\left(e_{\psi}\right)
$$

with the update laws given by $\dot{\hat{K}}_{e}^{T}=-\Gamma_{e}\left(x e-\sigma_{e} \hat{K}_{e}\right)$ and $\dot{\hat{\Theta}}=\Gamma_{\theta}\left(\Phi(x) e-\sigma_{\theta} \hat{K}_{\theta}\right)$.

Table IV shows the values of $\Gamma_{e, \theta}$ and $\sigma_{e, \theta}$ chosen for this controller. These values were found through iteration, with emphasis on enhancing long-term tracking of the desired trajectory.

TABLE IV: ADAPTIVE CONTROLLER GAINS AND LEAKAGE TERMS

\begin{tabular}{|l|c|c|} 
& $e$ & $\Theta$ \\
\hline$\Gamma$ & 0.15 & 0.22 \\
\hline$\sigma$ & 0.05 & 0.02 \\
\hline
\end{tabular}

\section{E. Fixed-gain PID Controller with Adaptive Augmentation}

This controller is a hybrid between the fixed-gain PID controller (Section III.B) and the adaptive PID controller (Section III.C). This scheme uses the fixed-gain PID controller as a baseline control and has the adaptive PID control to augment it to enhance robustness. An application of such a system is found in [9] to improve accuracy and speed of a laser scanner process. The baseline PID control is as described in Section III.B, with the same following control equations:

$$
u_{\psi}^{P I D}=K_{P}^{b} e_{\psi}+K_{I}^{b} \int e_{\psi}+K_{D}^{b} \dot{e}_{\psi}
$$

where $e_{\psi}=\psi_{\text {actual }}-\psi_{d}$ and $\dot{e}_{\psi}=\dot{\psi}_{\text {actual }}-\dot{\psi}_{d}$.

This PID controller is augmented with an adaptive component. Adaptive augmentation adds adaptive gains to the fixed gains of the PID mentioned above with the goal of improving tracking of the desired trajectory [10] and making the closed loop system more robust. Note that in this application, only proportional and derivative terms feature augmentation; the integral term is still a fixed gain. The adaptive portion of the control law that is used to augment the fixed gain PID controller is:

$$
u_{\psi}^{A D}=g_{P}^{A D} e_{\psi}+g_{D}^{A D} \dot{e}_{\psi}
$$

with the derivative of the adaptive control gains calculated by $\dot{g}_{P}^{A D}=-\Gamma_{P} e_{\psi}{ }^{2}+\sigma_{1} g_{P}$ and $\dot{g}_{D}^{A D}=-\Gamma_{D} e_{\psi} \dot{e}_{\psi}+\sigma_{3} g_{D}$.

Summing the adaptive and fixed gain control signals gives the total control command:

$$
u_{\psi}^{T O T A L}(t)=g_{P}^{T O T} e_{\psi}(t)+g_{I}^{T O T} \int e d t+g_{D}^{T O T} \dot{e}_{\psi}(t),
$$

where the total gains are defined by $g_{P}^{T O T}=\left(K_{P}^{b}+g_{P}^{A D}\right), g_{I}^{T O T}=K_{I}^{b}$ and $g_{D}^{T O T}=\left(K_{D}^{b}+g_{D}^{A D}\right)$.

Each of the values represented by $K_{P, I, D}^{b}$ is a fixed-gain value for the baseline PID controller. Each $\Gamma_{1,3}$ value represents adaptive gain multipliers, while each $\sigma_{1,3}$ determines the emphasis on the sigma modification term used to prevent wind-up. Also, a saturation limiter was used on the adaptive gains so that they did not rise too high, setting the maximum adaptive gains to $\pm 50 \%$ of the value of the fixed gains. The values for $\Gamma_{P, D}, K_{P, I, D}$, and $\sigma_{P, D}$ can be found in Table $\mathrm{V}$ and are the same as those used in sections III-B and III-C.

TABLE V: PID WITH ADAPTIVE AUGMENTATION CONTROLLER GAINS AND LEAKAGE TERMS

\begin{tabular}{|c|c|c|c|} 
& Proportional & Integral & Derivative \\
\hline$\Gamma$ & -.0 .2 & -- & 12 \\
\hline$K$ & 0.5 & 0.006 & 2 \\
\hline$\sigma$ & -0.035 & -- & -0.06 \\
\hline
\end{tabular}

IV. RESULTS

Three sets of simulations are run to evaluate the performance of the developed controllers. The fist simulation set is run with a constant desired heading of $90^{\circ}$. This test is used to evaluate the performance of the four second layer controllers alone, which seek to eliminate heading error. The second set of simulations is used to quantify the ability of the combined first and second layer control algorithms to eliminate across-track error when subject to disturbances. This simulation is performed at the input thrust of $2000 \mathrm{~N}$ per engine, which is the input thrust for which the controllers were tuned. The third set of simulations is similar to the second set with the exception that the forward thrust of each engine is reduced from $2000 \mathrm{~N}$ to $1200 \mathrm{~N}$. This is done to test the robustness of the developed controllers to changes in operating inputs. 


\section{A. Fixed Desired Heading}
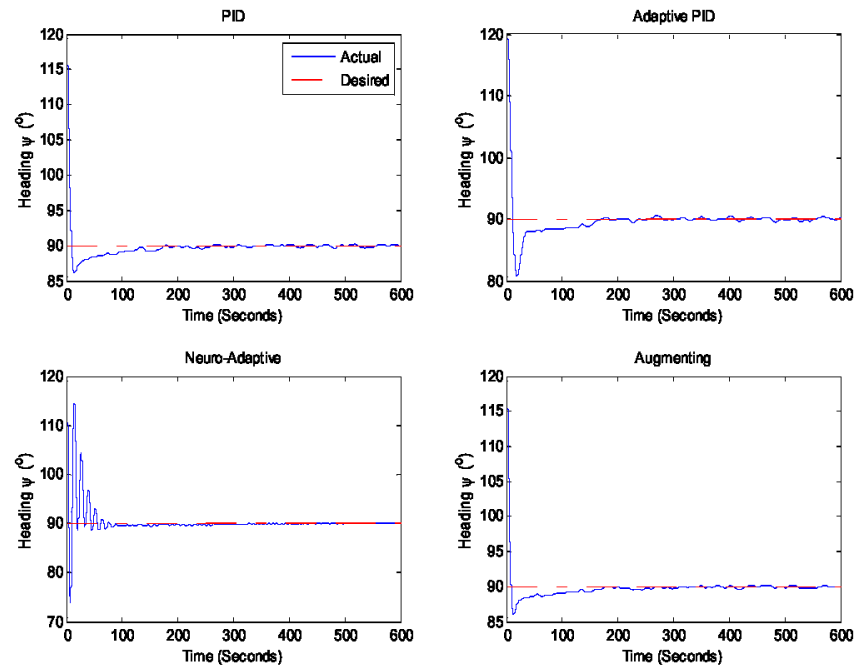

Figure 1: Plots of each controller's following to a constant heading over time
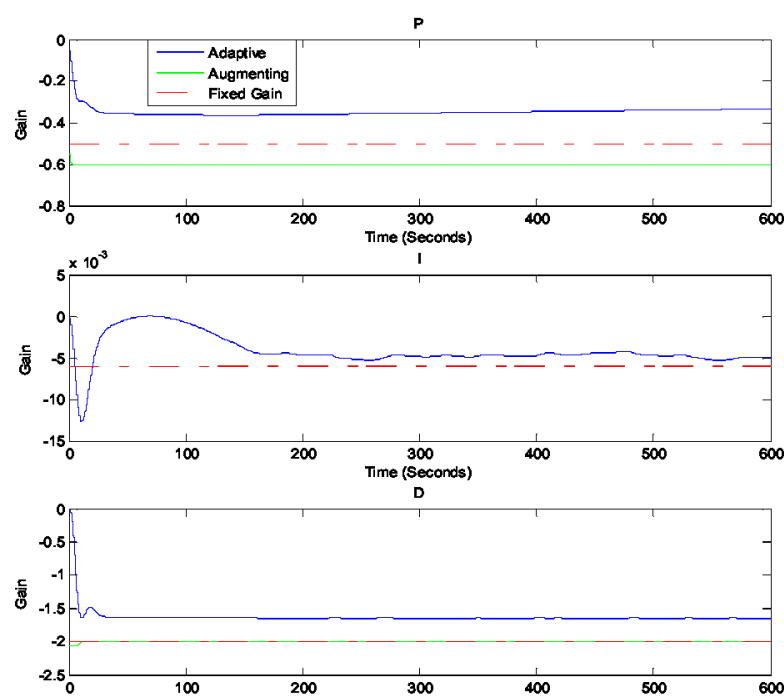

Figure 2: Plots of gains over Time following constant heading

First, the results from each second-layer controller will be shown for their ability to follow a constant value for heading. This is a feature common to most autopilots and provides an opportunity to analyze each second layer controller. For this test, the constant heading value chosen is $90^{\circ}$ (perpendicular to the environmental disturbances), while the initial heading is $120^{\circ}$.

Figure 1 shows how each controller follows a constant heading, in this case $90^{\circ}$, while Figure 2 presents $\mathrm{P}$, I, and D gains for the controllers and Figure 3 shows the control command for each controller.

During the initial tuning phase where the vessel starts with an initial heading error of $30^{\circ}$, all four controllers force the vessel to reach the desired heading in less than $11 \mathrm{~s}$, before overshooting the desired heading (Figure 1). The three PID controllers then converge towards the desired heading relatively smoothly with the adaptive augmenting controller having the smallest overshoot and fastest convergence rate. Conversely, the neuro-adaptive controller oscillates about the desired heading several times with oscillation amplitudes of up to $24^{\circ}$ before converging on the desired heading after about $90 \mathrm{~s}$. During this initial phase the neuro-adaptive controller varies the engine angle by up to $30^{\circ}$, while the fixed gain PID and adaptive augmenting PID use engine angles of up to $15^{\circ}$ and the adaptive PID uses a maximum engine angle of only $5^{\circ}$.

After the initial transient tuning phase of approximately $100 \mathrm{~s}$, each controller is able to achieve nearly a constant heading with all controllers holding the magnitude of the heading error below $2^{\circ}$ after the first $100 \mathrm{~s}$ and $0.5^{\circ}$ after the first $300 \mathrm{~s}$. The neuro-adaptive controller has the smallest heading error standard deviation of only $0.0672^{\circ}$ after the first $300 \mathrm{~s}$ while the adaptive PID controller has the largest heading error for the last $300 \mathrm{~s} \mathrm{of} 0.2166^{\circ}$. This performance is achieved for the last $300 \mathrm{~s}$ using engine angles with a standard deviation of $0.085^{\circ}$ for the fixed gain PID, $0.092^{\circ}$ for the adaptive augmenting PID, $0.097^{\circ}$ for the adaptive PID, and $0.269^{\circ}$ for the neuro-adaptive controllers. During these simulations the adaptive PID gains are the lowest set of PID gains with the exception of the integral term near the beginning of the simulation. The adaptive augmenting integral and derivative gains are very near those of the fixed gain PID controller during the entire simulation while the proportional gain for the adaptive augmenting controller is significantly larger than the equivalent fixed PID gain for the entire simulation. All of the adaptive gains converge to nearly-constant values long before the end of this $600 \mathrm{~s}$ simulation. Table VI gives a side-by-side comparison of the standard deviation of the heading error and control signal during the last $300 \mathrm{~s}$ of the simulation.
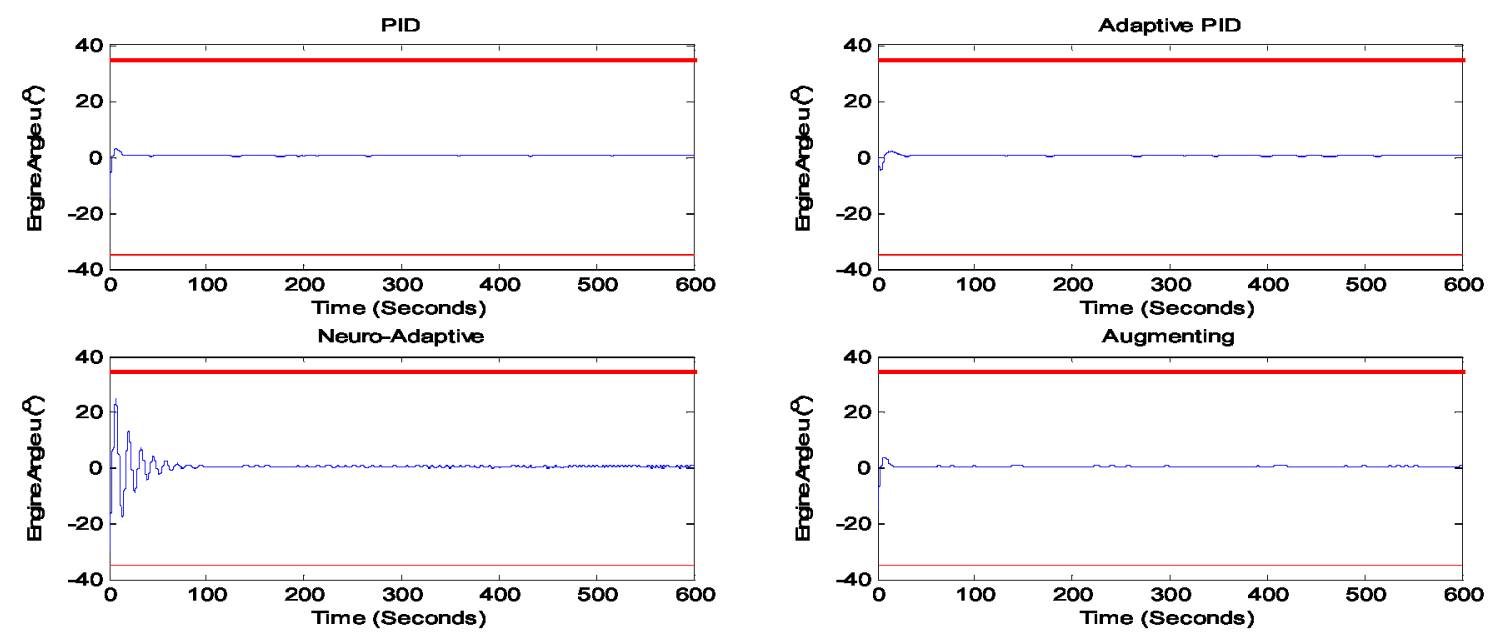

Figure 3: Plots of Control Signal (Engine Angle) for each controller following constant heading 

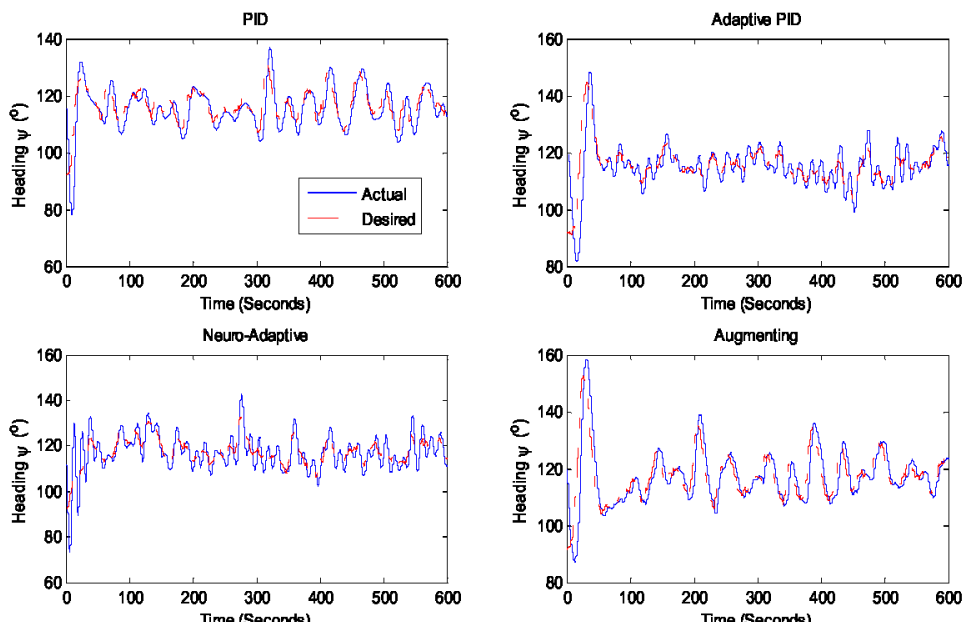

Figure 4: Plot of Heading and Desired Heading, $2^{\text {nd }}$ Layer, 2000 N Thrust
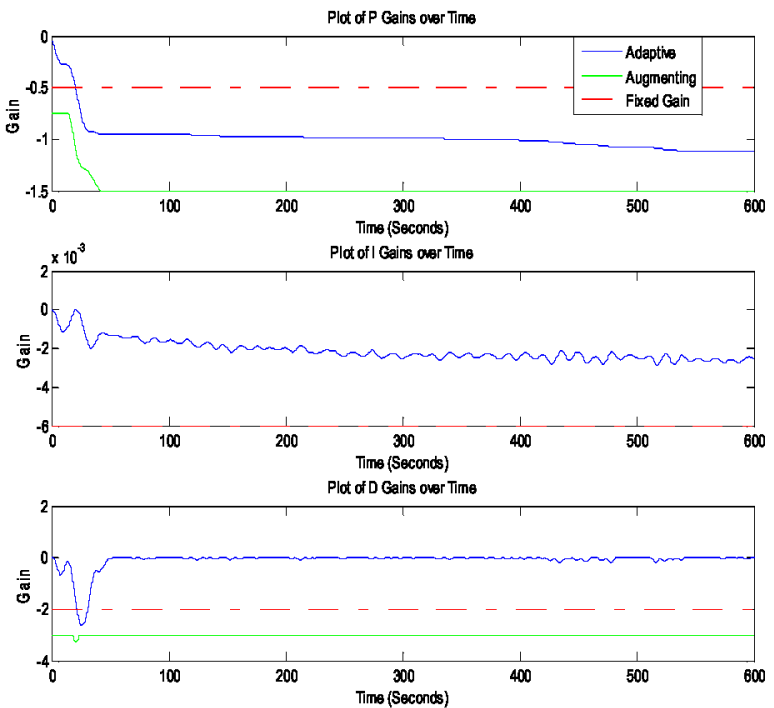

Figure 5: Plot of Gains for $2^{\text {nd }}$ Layer Controller, 2000N Thrust
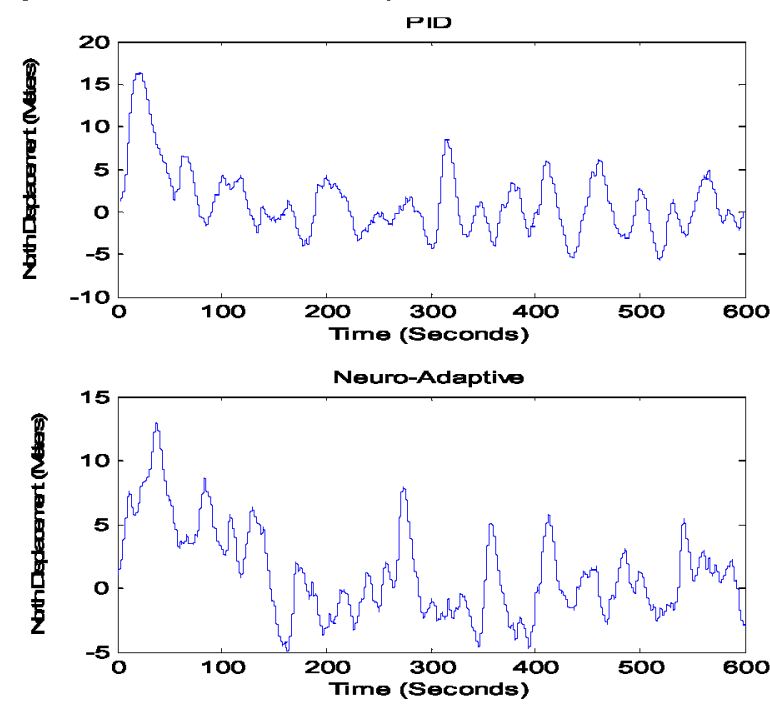

Next, each controller was tasked to follow the desired due East course in the presence of the environmental disturbances described in Section II. Figure 4 presents actual and desired heading, Figure 5 shows the controller gains, Figure 6 shows Engine Angle, and Figure 7 shows north displacement. As before, each simulation is run with initial heading of $120^{\circ}$.

The fixed gain PID Controller had good tracking of the desired heading specified by the first layer controller during this simulation. However, it was out-performed by all of the adaptive controllers for minimizing heading error during the second $300 \mathrm{~s}$ of the simulation with a standard deviation for heading of $4.99^{\circ}$. It also
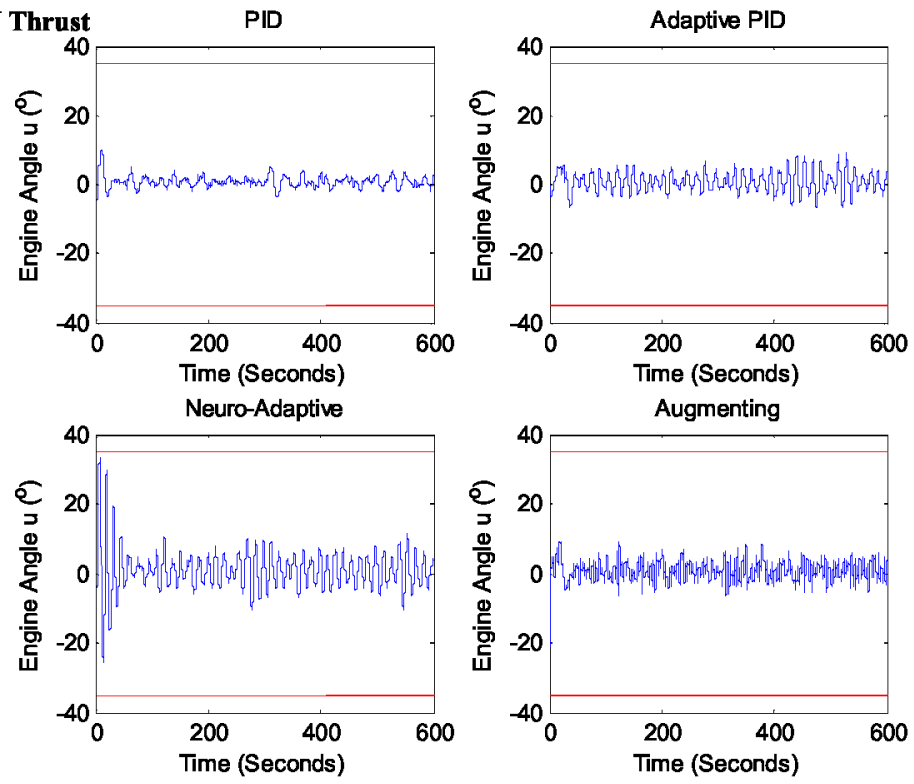

Figure 6: Plot of Control Signals over Time, $2^{\text {nd }}$ Layer, 2000N Thrust
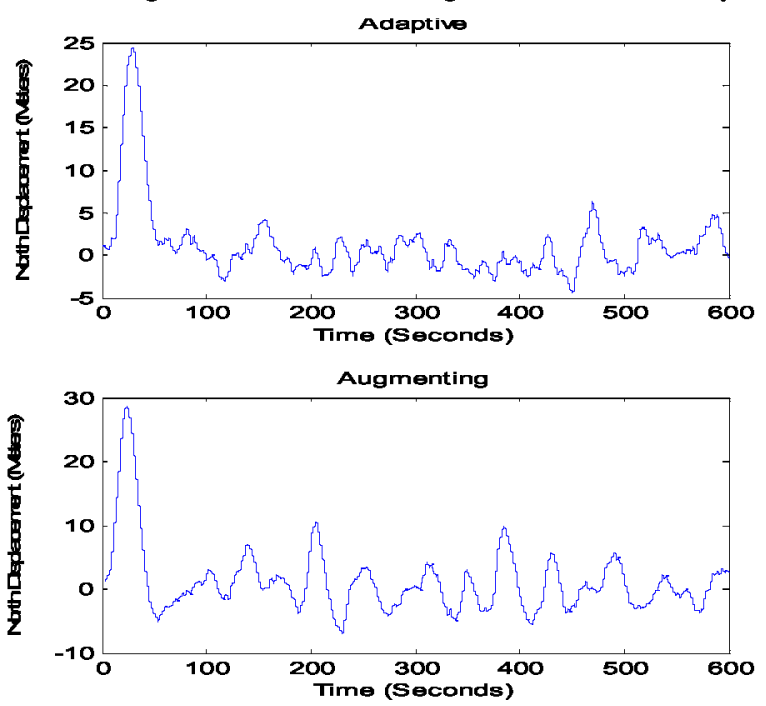

Figure 7: Plot of North Displacement over Time, $2^{\text {nd }}$ Layer, 2000 N Thrust 
had the largest across-track error with a standard deviation of $3.09 \mathrm{~m}$. The adaptive PID controller did the best job of minimizing the across-track error during the second $300 \mathrm{~s}$ of the simulation with a standard deviation of only $2.07 \mathrm{~m}$. The neuro-adaptive had slightly greater across track error during the second $300 \mathrm{~s}(2.28 \mathrm{~m}$ standard deviation) but had multiple heading oscillations not seen when the other three controllers are used, which converge after about $60 \mathrm{~s}$. This is also seen in the control signal, where the neuro-adaptive has signals of about $\pm 30^{\circ}$, while the other 3 controllers had control signals in the range of $\pm 5^{\circ}$ over the entire simulation. The augmenting controller had a slightly larger across-track error than the other two adaptive controllers during the second $300 \mathrm{~s}$ of the simulation $(2.87 \mathrm{~m}$ standard deviation) but during the same time period had the best in following the desired heading $\left(2.98^{\circ}\right.$, standard deviation). The augmenting controller also had the lowest gains of the 3 PID-based controllers, while the adaptive and fixed-gain controllers were larger, but the controllers had gains that were close in relation to one another. Further quantification of these values over the final $300 \mathrm{~s}$ is also found in Table VI.

\section{Following a straight line with $1200 \mathrm{~N}$ thrust per engine}

Operating conditions are never constant and survey speeds often vary for different operations. With this in mind, one more comparison of the controllers was performed. In this test, the same environmental conditions were used as for the previous tests, but the thrust for each motor was reduced from $2000 \mathrm{~N}$ to $1200 \mathrm{~N}$. After making this change, one can see the PID controller performance in an environment separate from the one in which it was tuned degraded significantly, while the adaptive controllers adjusted to the new operating conditions.

As in sections IV.A and IV.B, the initial heading for each test is $120^{\circ}$ with a desired path being an eastward trajectory. With this deviation from the conditions for which it was tuned, the performance of the fixed-gain PID controller deteriorates. As Table VI shows, the fixed-gain PID controller now has the worst performance in standard deviation of North error $(4.8379 \mathrm{~m})$ and heading error $\left(10.2831^{\circ}\right)$. This gives an increase in heading error of almost $6^{\circ}$ for the PID. Meanwhile, the performance of the adaptive controllers stayed relatively consistent with each adaptive controller having an increase in North error of no more than $0.4 \mathrm{~m}$ and increase in heading error of no more than $0.4^{\circ}$. In addition, the augmenting controller's North error improved from the test in Section IV.B. The fixed gain PID also had the largest increase in control signal, yet still has the lowest control signal standard deviation at $2.1695^{\circ}$. The neuro-adaptive controller still has the transient period in the beginning with large oscillations and control signal on the order of $\pm 30^{\circ}$, but ends up having the best performance in minimizing North error, although its heading error is higher than that of the adaptive PID and augmenting controller.

The PID controller appears sluggish in following the desired heading. This can be because with decreased thrust, the vessel does not rotate as quickly to a given engine angle. In this case, the vessel only has $60 \%$ of the thrust for which it is tuned for, and can not adapt to this change in thrust. Thus, as shown in Figure 8, there is a $15 \mathrm{~s}$ bias between the vessel's desired heading and the actual heading.
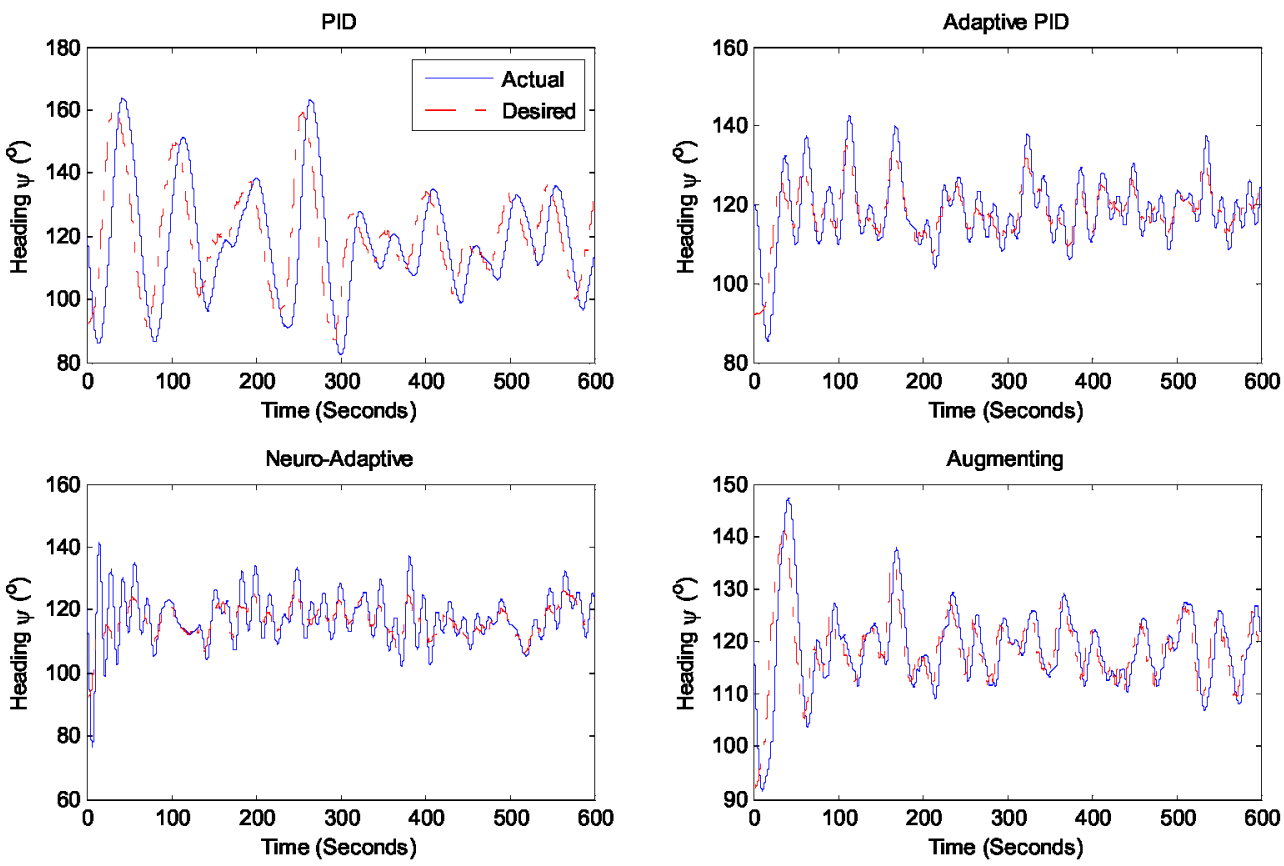

Figure 8: Plot of actual and desired heading, for 1200 N Constant Thrust 

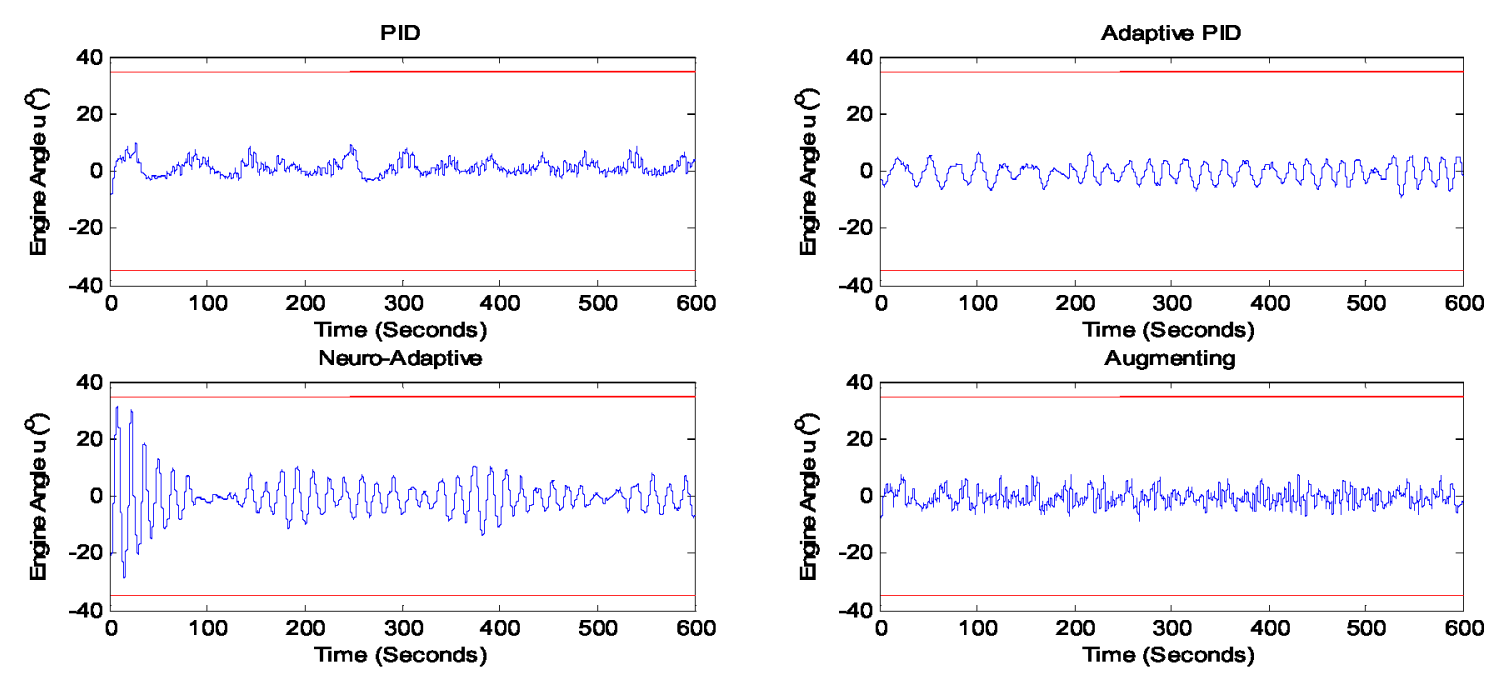

Figure 9: Plot of control signal over time, 1200 N Constant Thrust
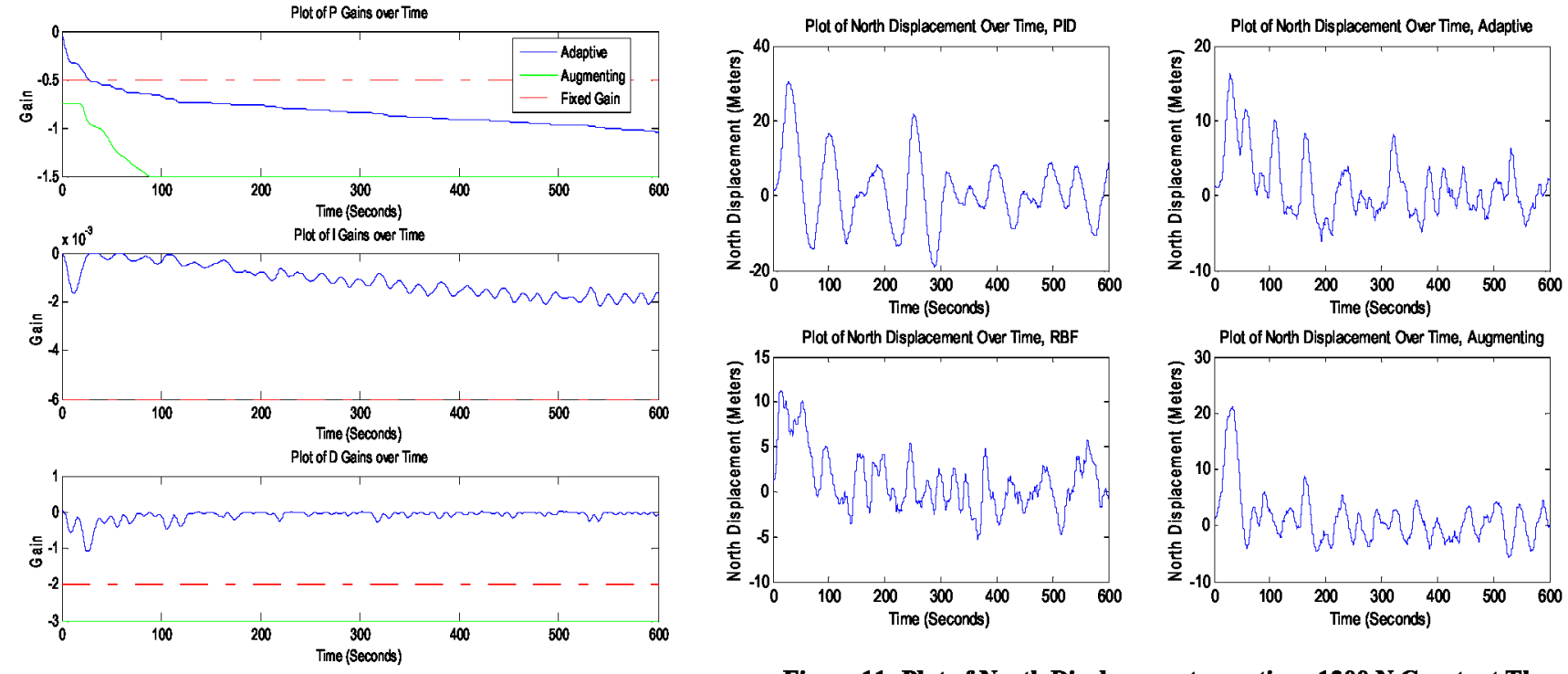

Figure 10: Plot of Gains over time, 1200 N Constant Thrust

Figure 11: Plot of North Displacement over time, 1200 N Constant Thrust

\section{Results Summary}

As can be seen in Table VI, the adaptive controllers out-perform their fixed-gain counterpart. The PID controller performs comparably to the adaptive controllers when following a fixed heading in the presence of environmental disturbances, even outperforming the adaptive PID in this test. However, its performance diminishes when following a variable heading as seen in Sections IV.B and IV.C. In Section IV.B the PID controller has the worst performance but its North error is relatively close to the augmenting controllers'. Meanwhile, the adaptive PID has the best performance in terms of North error, while the augmenting controller minimizes heading error the best.

When thrust is reduced down to $1200 \mathrm{~N}$, it can be seen that the performance of the PID controller is by far the worst. Meanwhile, the adaptive controllers have relatively similar performance to that of Section IV.B. The augmenting controller's North error even decreases by almost $0.4 \mathrm{~m}$. The heading error for each adapting controller increases, although not as much as the fixed-gain PID. The control signal also increased for the adaptive PID and the neuro-adaptive controllers, although these values remain small relative to the range $\left( \pm 35^{\circ}\right)$ of control signals that the steering system can accept. 
Table VI: Quantization of Errors in vessel simulations over the final 300 seconds

\begin{tabular}{|l|c|c|c|c|c|c|c|c|}
\hline & \multicolumn{3}{|c|}{ Standard Deviation of North and Heading Errors and Control Signal over Final 300 Seconds } \\
\hline & \multicolumn{2}{|c|}{ Constant Heading } & \multicolumn{3}{|c|}{ 2-layer, 2000 N } & \multicolumn{3}{c|}{ 2-Layer, 1200 N } \\
\hline & $\begin{array}{c}\text { Heading } \\
\text { Error }\end{array}$ & $\begin{array}{c}\text { Control } \\
\text { Signal }\end{array}$ & $\begin{array}{c}\text { North } \\
\text { Error }\end{array}$ & $\begin{array}{c}\text { Heading } \\
\text { Error }\end{array}$ & $\begin{array}{c}\text { Control } \\
\text { Signal }\end{array}$ & $\begin{array}{c}\text { North } \\
\text { Error }\end{array}$ & $\begin{array}{c}\text { Heading } \\
\text { Error }\end{array}$ & $\begin{array}{c}\text { Control } \\
\text { Signal }\end{array}$ \\
\hline & Degrees & Degrees & Meters & Degrees & Degrees & Meters & Degrees & Degrees \\
\hline PID & 0.1371 & 0.0859 & 3.0902 & 4.4859 & 1.6159 & 4.8379 & 10.2831 & 2.1695 \\
\hline $\begin{array}{l}\text { Adaptive } \\
\text { PID }\end{array}$ & 0.2166 & 0.0974 & 2.0738 & 3.1761 & 3.2945 & 2.5638 & 3.7311 & 3.4077 \\
\hline $\begin{array}{l}\text { Neuro- } \\
\text { adaptive }\end{array}$ & 0.0672 & 0.2693 & 2.2853 & 3.9406 & 4.1826 & 2.3284 & 4.2975 & 4.5511 \\
\hline Augmenting & 0.1312 & 0.0917 & 2.8702 & 2.9821 & 2.7330 & 2.4877 & 3.3791 & 2.6379 \\
\hline
\end{tabular}

\section{CONCLUSION}

One fixed-gain and three adaptive controllers have been developed, tuned, and tested within a simulation for three separate cases to quantify performance in mitigating environmental forces acting upon a vessel. From the results of these tests, it can be seen that adaptive controllers are more robust in keeping a vessel on a desired path in the presence of nonlinear perturbations. Future work on this topic includes performing sea trials with these controllers, and possible comparison with commercially available autopilots.

\section{REFERENCES}

[1] "Marine Autopilots, Pick the Best." Marine Electronics Reviews. 14 August $2009<$ http://www.marine-electronics-reviews.com/marineautopilots.html>.

[2] Wing, Charlie. How Boat Things Work: An Illustrated Guide. McGraw-Hill, 2004.

[3] Fossen, T.I. Guidance and Control of Ocean Vehicles. New York: Wiley, 1994.

[4] Aidi, Shen and Chu Jianxin. Investigation of Ship Autopilot Based on FNN. The Eighth Conference on Electronic Measurement and Instruments, 1618 July 2007, 3-335-3-338.

[5] "Selecting a Raymarine Autopilot." West Marine: West Advisor. 14 August 2009 $<$ http:/www.westmarine.com/webapp/wcs/stores/servlet/westadvisor/10001/-1/10001/Autopilots.htm>.

[6] J.H. VanZwieten and F.R. Driscoll. (2008) "A comprehensive general simulation of twin screw displacement hull vessels with validation" Journal of Mathematical and Computer Modeling of Dynamical Systems, 14 (4) 269-301

[7] M.K. Ochi and Y.S. Shin, Wind turbulent spectra for design consideration of offshore structures. Proceedings of the Offshore Technology Conference, 2-5 May, Houston, Texas, 1988 pp.461-467.

[8] M.A. Donelan, J. Hamilton, and W.H. Hui, Directional spectra of wind-generated waves, Philos. Trans. R. Soc. Lond. A315 (1985), pp. 509-562.

[9] M. Lugmair et al. Result-Adaptive PID Control Improves Laserscanner Positioning. Baasel Lasertech.

[10] K. Hornik, M. Stinchcombe, H. White. Multilayer Feedforward Networks are Universal Approximators. IEE Transactions on Neural Networks 2 (1989) 359-366.

[11] Ioannou, Petros and Baris Fidan. Adaptive Control Tutorial. Philadelphia: SIAM, 2006.

[12] Lavretsky, Eugene. Adaptive Control: Introduction, Overview, and Applications. NASA Adaptive Control Workshop. NASA Marshall Space Center, Huntsville, AL. 24 Feb 2009.

[13] Zilouchian, Ali and Mo Jamshidi. Intelligent Control Systems Using Soft Computing Methodologies. New York: CRC Press, 2001

[14] Budny, Dan. Introduction to Engineering Analysis. Champaign: Stipes, 2003. 